\title{
Stronger Multi-Commodity Flow Formulations of the Capacitated Vehicle Routing Problem
}

\author{
Adam N. Letchford* Juan-José Salazar-González ${ }^{\dagger}$ \\ To appear in European Journal of Operational Research
}

\begin{abstract}
The Capacitated Vehicle Routing Problem is a much-studied (and strongly $\mathcal{N} \mathcal{P}$-hard) combinatorial optimization problem, for which many integer programming formulations have been proposed. We present two new multi-commodity flow (MCF) formulations, and show that they dominate all of the existing ones, in the sense that their continuous relaxations yield stronger lower bounds. Moreover, we show that the relaxations can be strengthened, in pseudo-polynomial time, in such a way that all of the so-called knapsack large multistar (KLM) inequalities are satisfied. The only other relaxation known to satisfy the KLM inequalities, based on set partitioning, is strongly $\mathcal{N} \mathcal{P}$-hard to solve. Computational results demonstrate that the new MCF relaxations are significantly stronger than the previously known ones.
\end{abstract}

Keywords: vehicle routing, cutting planes, multi-commodity flows, integer programming.

\section{Introduction}

Vehicle Routing Problems (VRPs) are classic problems in operational research and logistics, and have also received a great deal of attention from the combinatorial optimization community. A huge number of papers have been written on the theory and applications of VRPs, and on exact and heuristic solution methods for them (see, e.g., the edited volumes $[5,14,27]$.)

This paper is concerned with the Capacitated VRP (CVRP), which Dantzig and Ramser [7] defined as follows. A fleet of identical vehicles, with limited capacity, is located at a depot. There are $n$ customers that require service. Each customer has a known demand. The cost of travel between any pair of customers, or between any customer and the depot, is also known. The task is to find a minimum-cost collection of vehicle routes,

\footnotetext{
* Department of Management Science, Lancaster University, Lancaster LA1 4YW, U.K.

${ }^{\dagger}$ DEIOC, Universidad de La Laguna, 38271 Tenerife, Spain
} 
each starting and ending at the depot, such that each customer is visited by exactly one vehicle, and no vehicle visits a set of customers whose total demand exceeds the vehicle capacity.

Letchford \& Salazar [22] surveyed and compared several integer programming formulations of the CVRP. These included the so-called two- and three-index formulations, the single-, two- and multi-commodity flow formulations, and the set partitioning formulations. At present, the most successful exact algorithms for the CVRP are based on the two-index formulation (e.g., Lysgaard et al. [23]) or on set partitioning formulations (e.g., Fukasawa et al. [10], Baldacci et al. [3]).

One way to measure the strength of an alternative formulation is to project the feasible region of its continuous relaxation into the space of the natural (two-index) formulation. Gouveia [15] showed that, in the case of the single-commodity flow formulation, the projection satisfies a family of valid inequalities now known as generalized large multistar (GLM) inequalities. Letchford \& Salazar [22] showed that the projection of the set partitioning formulation (with only elementary routes permitted) satisfies the so-called knapsack large multistar (KLM) inequalities, defined in [21]. The KLM inequalities include the GLM inequalities and the so-called subtour elimination (SE) inequalities as special cases. Unfortunately, the continuous relaxation of the set partitioning formulation is itself strongly $\mathcal{N} \mathcal{P}$-hard to solve.

This paper has four main contributions. First, we show how to strengthen the two best multi-commodity flow ( $\mathrm{MCF}$ ) formulations, by adding only a polynomial number of additional constraints. Second, we show that the projections of our two formulations satisfy the GLM and SE inequalities. Third, we show that the new formulations can be further strengthened, in pseudo-polynomial time, in such a way that all of the KLM inequalities are satisfied. (We remark that no polynomial or pseudo-polynomial time separation algorithm is known for the KLM inequalities themselves.) Finally, we present some computational results that demonstrate that the new MCF formulations are significantly stronger than the previously known ones.

As mentioned above, the current best algorithms for the CVRP are based on two-index or set partitioning formulations. The contribution of this paper may therefore appear to be only of theoretical interest. We would like to point out, however, that there exist variants of the CVRP for which it is natural, or even essential, to use additional commodity-flow variables. This includes, for example, the problem described in in [17], in which several distinct products have to be picked up and delivered at various locations, and the one described in [19], in which the cost of traversing an arc is an increasing function of vehicle load. Potentially, our results could be used to derive better formulations and algorithms for such problems.

The structure of the paper is as follows. The literature is reviewed in Section 2. The strengthened MCF formulations are presented and analysed in Section 3. The result on KLM inequalities is given in Section 4. Some 
computational results are given in Section 5, and some concluding remarks are made in Section 6.

Throughout the paper, we use the following notation. We have a complete directed graph $G$ with node set $V=\{0,1, \ldots, n\}$ and arc set $A$. Node 0 represents the depot, and nodes $1, \ldots, n$ represent customers. We sometimes write $V_{c}$ for $V \backslash\{0\}$, the set of customer nodes. The (positive integer) demand of customer $i \in V_{c}$ is $q_{i}$. The (positive integer) vehicle capacity is $Q$. The (non-negative integer) cost of traversing arc $(i, j) \in A$ is $c_{i j}$. (Our approach can easily be adapted to the case of symmetric costs and/or the case in which the number of vehicles is restricted.)

\section{Literature Review}

As mentioned above, many formulations have been proposed for the CVRP. For brevity, we review only ones of relevance here. Subsections 2.1 to 2.4 cover two-index vehicle flow, single- and two-commodity flow, multicommodity flow and set partitioning formulations, respectively.

\subsection{The two-index vehicle flow formulation}

Laporte \& Nobert [20] presented what is now called the two-index vehicle flow formulation. For all $(i, j) \in A$, define a binary variable $x_{i j}$, taking the value 1 if and only if some vehicle travels from $i$ to $j$. For any $S \subset V$, let $\delta^{+}(S)$ (respectively, $\delta^{-}(S)$ ) denote the set of $\operatorname{arcs}(i, j)$ with $i \in S, j \in V \backslash S$ (respectively, with $i \in V \backslash S, j \in S$ ). If $S=\{i\}$ then we will write $\delta^{+}(i)$ and $\delta^{-}(i)$ rather than $\delta^{+}(\{i\})$ and $\delta^{-}(\{i\})$, for brevity. Given some $F \subset A$, let $x(F)$ denote $\sum_{(i, j) \in F} x_{i j}$. Finally, for any set of customers $S \subset V_{c}$, let $q(S)=\sum_{i \in S} q_{i}$. Then the formulation is:

$$
\begin{array}{ccl}
\min & \sum_{(i, j) \in A} c_{i j} x_{i j} & \\
\text { s.t. } & x\left(\delta^{+}(i)\right)=1 & \left(i \in V_{c}\right) \\
& x\left(\delta^{-}(i)\right)=1 & \left(i \in V_{c}\right) \\
x\left(\delta^{+}(S)\right) \geq\lceil q(S) / Q\rceil & \left(S \subseteq V_{c}\right) \\
& x_{i j} \in\{0,1\} & ((i, j) \in A) .
\end{array}
$$

The out-degree equations (2) and the in-degree equations (3) ensure that vertices are visited exactly once. The constraints (4), called rounded capacity (RC) inequalities, prevent the existence of infeasible routes, and also have the side-effect of preventing subtours. Finally, (5) are the integrality conditions on the $x$-variables.

Several families of valid linear inequalities (cutting planes) have been developed for the two-index vehicle flow formulation (see Naddef \& Rinaldi [25] for a survey). We will be interested in the following inequalities: 
- The fractional capacity (FC) inequalities:

$$
x\left(\delta^{+}(S)\right) \geq \frac{q(S)}{Q} \quad\left(S \subseteq V_{c}\right) .
$$

- The subtour elimination (SE) inequalities:

$$
x\left(\delta^{+}(S)\right) \geq 1 \quad\left(S \subseteq V_{c}\right) .
$$

- The generalized large multistar (GLM) inequalities (see Gouveia [15]):

$$
x\left(\delta^{+}(S)\right) \geq \frac{1}{Q} \sum_{i \in S}\left(q_{i}+\sum_{j \in V_{c} \backslash S} q_{j}\left(x_{i j}+x_{j i}\right)\right) \quad\left(S \subseteq V_{c}\right) .
$$

- The knapsack large multistar (KLM) inequalities (see Letchford et al. [21]):

$$
x\left(\delta^{+}(S)\right) \geq \frac{1}{\beta} \sum_{i \in S}\left(\alpha_{i}+\sum_{j \in V_{c} \backslash S} \alpha_{j}\left(x_{i j}+x_{j i}\right)\right) \quad\left(S \subseteq V_{c}\right),
$$

where $\alpha \geq 0$ and $\beta>0$ are such that the inequality $\sum_{i \in V_{c}} \alpha_{i} y_{i} \leq \beta$ is valid for the $0-1$ knapsack polytope:

$$
K P(Q, q):=\operatorname{conv}\left\{y \in\{0,1\}^{n}: \sum_{i \in V_{c}} q_{i} y_{i} \leq Q\right\} .
$$

Obviously, the $\mathrm{RC}$ inequalities dominate the $\mathrm{FC}$ and $\mathrm{SE}$ inequalities, and the GLM inequalities dominate the FC inequalities. It is also not difficult to see that the KLM inequalities include the GLM and SE inequalities as special cases. In general, there are no other dominance relations.

\subsection{Single- and two-commodity flow formulations}

The first single-commodity flow formulation, that we call "SCF1", was presented by Gavish \& Graves [12]. A continuous variable $f_{i j}$ is defined for each $(i, j) \in A$, representing the total load (if any) carried along the arc $(i, j)$. One then replaces constraints (4) in the two-index vehicle flow formulation with:

$$
\begin{aligned}
f\left(\delta^{-}(i)\right)-f\left(\delta^{+}(i)\right)=q_{i} & \left(i \in V_{c}\right) \\
0 \leq f_{i j} \leq Q x_{i j} & ((i, j) \in A) .
\end{aligned}
$$

The constraints (11) ensure that each customer $i$ receives the demand of $q_{i}$. The constraints (12) are just bounds on the $f$ variables. 
Gavish [13] proposed to strengthen SCF1 by replacing the bounds (12) with the stronger bounds

$$
q_{j} x_{i j} \leq f_{i j} \leq\left(Q-q_{i}\right) x_{i j} \quad((i, j) \in A) .
$$

We call this strengthened formulation "SCF2".

Gouveia [15] used Hoffman's circulation theorem [18] to project the feasible regions of the LP relaxations of SCF1 and SCF2 into the space of the $x$ variables. The projection of SCF1 is given by the out-degree equations (2), the in-degree equations (3), the FC inequalities (6) and non-negativity. The projection of SCF2, as expected, is stronger, satisfying the GLM inequalities (8) in place of the FC inequalities.

In Baldacci et al. [2], a two-commodity flow formulation is presented for the case in which the $\operatorname{costs} c_{i j}$ are symmetric. Letchford \& Salazar [22] show that, in this case, the LP relaxation of their formulation gives the same lower bound as that of SCF2.

\subsection{Multi-commodity flow formulations}

The first multi-commodity flow formulation, that we call "MCF1a", was presented in Garvin et al. [11]. A binary variable $f_{i j}^{k}$ is defined for each $k \in V_{c}$ and each $(i, j) \in A$, taking the value 1 if and only if a vehicle traverses $(i, j)$ on the way from the depot to $k$. The formulation is then obtained by replacing constraints (4) in the two-index vehicle flow formulation with:

$$
\begin{aligned}
f^{k}\left(\delta^{+}(0)\right)=f^{k}\left(\delta^{-}(k)\right)=1 & \left(k \in V_{c}\right) \\
f^{k}\left(\delta^{-}(0)\right)=f^{k}\left(\delta^{+}(k)\right)=0 & \left(k \in V_{c}\right) \\
f^{k}\left(\delta^{+}(i)\right)=f^{k}\left(\delta^{-}(i)\right) & \left(k, i \in V_{c}: i \neq k\right) \\
f_{i j}^{k} \geq 0 & \left(k \in V_{c},(i, j) \in A\right) \\
\sum_{k \in V_{c}} q_{k} f_{i j}^{k} \leq Q x_{i j} & ((i, j) \in A) .
\end{aligned}
$$

The single-source multi-commodity flow theorem of Papernov [26] implies that the LP relaxations of MCF1a and SCF1 are of equal strength.

Gavish [13] proposed an alternative formulation, that we call "MCF1b". It is obtained by replacing (17) with the following constraints:

$$
\begin{aligned}
\sum_{k \in V_{c} \backslash\{i\}} q_{k} f^{k}\left(\delta^{+}(i)\right) \leq Q-q_{i} \quad\left(i \in V_{c}\right) \\
f_{i j}^{k} \leq x_{i j} \quad\left(k \in V_{c},(i, j) \in A\right) .
\end{aligned}
$$

It follows from the max-flow/min-cut theorem that, if the constraints (18) are dropped from MCF1b, then the projection into $x$-space is given by (2), (3), (7) and non-negativity. No similar projection result is known for MCF1b itself. 
Letchford \& Salazar [22] presented a different MCF formulation, with two commodities per customer. For each arc $(i, j)$ and customer $k$, the variable $f_{i j}^{k}$ is defined as before, but there is now also a binary variable $g_{i j}^{k}$, taking the value 1 if and only if a vehicle traverses $(i, j)$ on the way from $k$ to the depot. We then replace the constraints (4) in the two-index vehicle flow formulation with the constraints (13)-(16), together with:

$$
\begin{aligned}
g^{k}\left(\delta^{+}(k)\right)=g^{k}\left(\delta^{-}(0)\right)=1 & \left(k \in V_{c}\right) \\
g^{k}\left(\delta^{-}(k)\right)=g^{k}\left(\delta^{+}(0)\right)=0 & \left(k \in V_{c}\right) \\
g^{k}\left(\delta^{+}(i)\right)=g^{k}\left(\delta^{-}(i)\right) & \left(k, i \in V_{c}: i \neq k\right) \\
g_{i j}^{k} \geq 0 & \left(k \in V_{c},(i, j) \in A\right) \\
f_{i j}^{k}+g_{i j}^{k} \leq x_{i j} & \left(k \in V_{c},(i, j) \in A\right) \\
\sum_{k \in V_{c} \backslash\{i\}} q_{k}\left(f^{i}\left(\delta^{+}(k)\right)+g^{i}\left(\delta^{-}(k)\right)\right) \leq Q-q_{i} & \left(i \in V_{c}\right) .
\end{aligned}
$$

We will call this formulation "MCF2a". Note that the depot is either the source or the sink of every commodity. The above-mentioned result by Papernov [26] then implies that, if the constraints (25) are dropped from MCF2a, then the projection into $x$-space is again given by $(2),(3),(7)$ and non-negativity. No similar projection result is known for MCF2a itself.

\subsection{Set partitioning formulations}

We will also need the following set partitioning (SP) formulation, due to Balinski \& Quandt [4]. Let $\Omega$ denote the set of possible routes for a single vehicle, and let $z_{r}$ for each $r \in \Omega$ be a binary variable taking the value 1 if and only if that route is used. Define the constant $a_{i r}$ for each customer $i$ and route $r$, taking the value 1 if $i$ is served by $r$, and 0 otherwise. Finally let $c_{r}$ denote the cost of route $r$. Then the SP formulation is:

$$
\begin{array}{lll} 
& \min \quad \sum_{r \in \Omega} c_{r} z_{r} & \\
\text { s.t. } & \sum_{r \in \Omega} a_{i r} z_{r}=1 & \left(i \in V_{c}\right) \\
& z_{r} \in\{0,1\} & (r \in \Omega) .
\end{array}
$$

Since the number of variables in this formulation can be exponential in $n$, column generation is necessary. Unfortunately, the pricing subproblem is easily shown to be strongly $\mathcal{N} \mathcal{P}$-hard. Agarwal et al. [1] solve it via integer programming. Foster \& Ryan [9] noted that pricing becomes easier if one enlarges the set $\Omega$ by allowing routes in which the vehicle is permitted to visit customers more than once (now called non-elementary routes). Pricing can then be performed in pseudo-polynomial time, by dynamic programming. See, e.g., Martinelli et al. [24] for details.

Letchford and Salazar [22] prove the following: 
- When elementary routes are used, the projection of the LP relaxation into $x$-space satisfies all KLM inequalities.

- Again, when elementary routes are used, the LP relaxation is at least as strong as those of all of the SCF and MCF formulations mentioned in the previous two subsections.

- If, however, non-elementary routes are permitted, then the only KLM inequalities which are satisfied by the projection are those in which $\alpha y \leq \beta$ is valid for the general integer knapsack polytope

$$
\operatorname{conv}\left\{y \in \mathbb{Z}_{+}^{n}: \sum_{i \in V_{c}} q_{i} y_{i} \leq Q\right\} .
$$

These less general KLM inequalities still include the GLM inequalities as a special case, but no longer include the SE inequalities.

\section{Stronger Multicommodity Flow Formulations}

In this section, we present four new multi-commodity flow formulations, each of which satisfies the SE inequalities (7) and GLM inequalities (8). The ones presented in Subsection 3.1 dominate SCF1, SCF2, MCF1a and MCF1b. The one presented in Subsection 3.2 also dominates MCF2a. The one presented in Subsection 3.3 is designed especially for instances with symmetric costs.

\subsection{Strengthening MCF1a and MCF1b}

In this subsection we will need the following lemma.

Lemma 1 The LP relaxation of formulation MCF1b satisfies the equations

$$
f_{i j}^{j}=x_{i j} \quad\left(j \in V_{c}, i \in V \backslash\{j\}\right) .
$$

Proof. Let $j \in V_{c}$ be fixed. From (3) we have $x\left(\delta^{-}(j)\right)=1$, from (13) we have $f^{j}\left(\delta^{-}(j)\right)=1$, and from (19) we have $f_{i j}^{j} \leq x_{i j}$ for all $i \in V \backslash\{j\}$. The only way for these to all hold simultaneously is for (26) to hold.

The following proposition introduces a class of valid inequalities.

Proposition 1 All (integer) solutions to formulation MCF1b satisfy the following inequalities:

$$
\sum_{k \in V_{c} \backslash\{i\}} q_{k} f_{i j}^{k} \leq\left(Q-q_{i}\right) x_{i j} \quad((i, j) \in A) .
$$

Proof. If the vehicle traverses the arc $(i, j)$, then it must have already delivered a demand of $q_{i}$ to customer $i$. 
Our first new formulation, which we call MCF1c, is obtained from MCF1b by replacing the constraints (18) with inequalities (27). The following two propositions state that MCF1c has some desirable properties.

Proposition 2 The LP relaxation of MCF1c satisfies the SE inequalities (7) and the GLM inequalities (8).

Proof. As mentioned in Subsection 2.3, the max-flow / min-cut theorem implies that the SE inequalities are satisfied. Now, for a given $S \subseteq V_{c}$ and a given commodity $k \in S$, the flow equations (13)-(15) imply that

$$
f^{k}\left(\delta^{-}(S)\right)=f^{k}\left(\delta^{+}(S)\right)+1 .
$$

Multiplying these equations by $q_{k}$ and summing over all $k \in S$, we obtain

$$
\sum_{k \in S} q_{k} f^{k}\left(\delta^{-}(S)\right)=\sum_{k \in S} q_{k} f^{k}\left(\delta^{+}(S)\right)+q(S) .
$$

Now, the constraints (27) for all $(i, j) \in \delta^{-}(S)$ imply that the left-hand side of (28) is no larger than

$$
\sum_{(i, j) \in \delta^{-}(S)}\left(Q-q_{i}\right) x_{i j}
$$

On the other hand, the constraints $(26)$ for all $(i, j) \in \delta^{+}(S)$, together with non-negativity, imply that the right-hand side of $(28)$ is no smaller than

$$
\sum_{(i, j) \in \delta^{+}(S)} q_{j} x_{i j}+q(S) .
$$

¿From this we deduce that the relaxation of MCF1d satisfies

$$
\sum_{(i, j) \in \delta^{-}(S)}\left(Q-q_{i}\right) x_{i j} \geq \sum_{(i, j) \in \delta^{+}(S)} q_{j} x_{i j}+q(S),
$$

which is equivalent to the GLM inequality (8) for the given $S$.

Proposition 3 The LP relaxation of MCF1C is stronger than that of SCF1, SCF2 and MCF1a, and at least as strong as that of MCF1b.

Proof. The LP relaxation of MCF1c satisfies the constraints (2), (3) (7) and (8). The fact that it is stronger than the LP relaxations of SCF1 and SCF2 then follow from the result of Gouveia [15] mentioned in Subsection 2.2. It is also stronger than the LP relaxation of MCF 1a since, as mentioned in Subsection 2.3, that relaxation is identical to the one of SCF1. To show that the LP relaxation of MCF1c is at least as strong as that of MCF1b, it 
suffices to show that it satisfies the inequalities (18). To this end, let $i \in V_{c}$ be fixed. Sum the inequalities (27) over all arcs entering $i$ to obtain

$$
\sum_{k \in V_{c}} q_{k} f^{k}\left(\delta^{-}(i)\right) \leq Q-\sum_{k \in V_{c} \backslash\{i\}} q_{k} x_{k i} .
$$

Together with the equation (13) for $i=k$, this implies

$$
\sum_{k \in V_{c} \backslash\{i\}} q_{k} f^{k}\left(\delta^{-}(i)\right) \leq Q-q_{i}-\sum_{k \in V_{c} \backslash\{i\}} q_{k} x_{k i} .
$$

The equations (15) then imply

$$
\sum_{k \in V_{c} \backslash\{i\}} q_{k} f^{k}\left(\delta^{+}(i)\right) \leq Q-q_{i}-\sum_{k \in V_{c} \backslash\{i\}} q_{k} x_{k i} .
$$

This dominates the inequality (18) for the given $i$.

Our computational results (Section 5) show that, in fact, the LP relaxation of MCF1c is stronger than that of MCF1b, and is also stronger than the relaxation in $x$-space defined by the out-degree equations (2), the indegree equations (3), the SE inequalities (7), the GLM inequalities (8), and non-negativity.

Now, in the proof of Proposition 3, we showed that the inequalities (18) are redundant, being implied by the other constraints in MCF1c. Interestingly, however, they can be strengthened (lifted) to obtain a non-redundant family of inequalities. These stronger inequalities are presented in the following proposition.

Proposition 4 All (integer) solutions to formulation MCF1c satisfy the following inequalities:

$$
\sum_{k \in V_{c} \backslash\{i\}} q_{k}\left(f^{i}\left(\delta^{+}(k)\right)+f^{k}\left(\delta^{+}(i)\right)\right) \leq Q-q_{i} \quad\left(i \in V_{c}\right) .
$$

Proof. Consider the vehicle that services customer $i$. The two terms on the left-hand side represent the total demand delivered by the vehicle before arriving at customer $i$ and after leaving customer $i$, respectively.

Adding the inequalities (29) to MCF1c, we obtain a formulation that we call "MCF1d". (For ease of reference, we present MCF1d in its entirety in the Appendix.) The computational results in Section 5 show that the LP relaxation of MCF1d is stronger than that of MCF1c, which shows that the inequalities (29) are not implied by the other linear constraints in MCF1d. 


\subsection{Strengthening MCF2a}

Now we turn our attention to MCF2a. We will show that MCF2a can be strengthened to obtain a formulation that also dominates MCF $1 d$, the strongest of the formulations given in the previous subsection. Our starting point is the following proposition:

Proposition 5 All (integer) solutions to formulation MCF2a satisfy the following constraints:

$$
f^{k}\left(\delta^{+}(i)\right)=g^{i}\left(\delta^{-}(k)\right) \quad\left(k, i \in V_{c}: i \neq k\right) .
$$

Proof. The left-hand and right-hand sides are each equal to 1 if customer $i$ is served before customer $k$ on the same route, and 0 otherwise.

The next step is to observe that, using Lemma 1, constraints (27) can be written as

$$
\sum_{k \in V_{c} \backslash\{i, j\}} q_{k} f_{i j}^{k} \leq\left(Q-q_{i}-q_{j}\right) x_{i j} \quad((i, j) \in A),
$$

The following proposition shows that these inequalities can then be strengthened, using the $g$-variables.

Proposition 6 All (integer) solutions to formulation MCF2a satisfy the following constraints:

$$
\sum_{k \in V_{c} \backslash\{i, j\}} q_{k}\left(f_{i j}^{k}+g_{i j}^{k}\right) \leq\left(Q-q_{i}-q_{j}\right) x_{i j} \quad((i, j) \in A) .
$$

Proof. If a vehicle traverses the $\operatorname{arc}(i, j)$, then it must have already delivered a demand of $q_{i}$ to customer $i$, and be about to deliver $q_{j}$ to customer $j$. Therefore the total load of the vehicle dedicated to the other customers on the route cannot exceed $Q-q_{i}-q_{j}$.

We now show that the constraints (25) can be discarded when (30) and (31) are considered. To this end, we need the following lemma:

Lemma 2 The LP relaxation of formulation MCF2a satisfies the equations

$$
f_{i j}^{j}=g_{i j}^{i}=x_{i j} \quad\left(i, j \in V_{c}: i \neq j\right) .
$$

Proof. Similar to the proof of Lemma 1.

Proposition 7 If the constraints (30) and (31) are added to MCF2a, then the constraints (25) become redundant. 
Proof. Use (21) and (32) to write (31) as:

$$
\sum_{k \in V_{c} \backslash\{i\}} q_{k}\left(f_{i j}^{k}+g_{i j}^{k}\right) \leq\left(Q-q_{i}\right) x_{i j} \quad((i, j) \in A) .
$$

Sum these over all $j \in V \backslash\{i\}$, and use (2) to obtain:

$$
\sum_{k \in V_{c} \backslash\{i\}} q_{k}\left(f^{k}\left(\delta^{+}(i)\right)+g^{k}\left(\delta^{+}(i)\right)\right) \leq Q-q_{i} \quad\left(i \in V_{c}\right) .
$$

Reverse the roles of $i$ and $k$, and use (15) and (30) to obtain (25).

Accordingly, we add constraints (30) and (31) to MCF2a, and delete the redundant constraints (25), to obtain what we call "MCF2b". (We present MCF $2 b$ in its entirety in the Appendix.) The following proposition shows that MCF 2b is the strongest of all the MCF formulations considered so far.

Proposition 8 Let $\left(x^{*}, f^{*}, g^{*}\right)$ be a solution to the LP relaxation of MCF2b. Then $\left(x^{*}, f^{*}\right)$ is a solution to the LP relaxation of MCF1d.

Proof. The feasible region of the LP relaxation of MCF1d is defined by the constraints (2), (3), (13)-(16), (19), (27) and (29). Constraints (2), (3) and (13)-(16) are already present in MCF2b. Constraints (19) are dominated by (24). Constraints (27) are dominated by (31). Finally, the fact that constraints (29) are satisfied by $\left(x^{*}, f^{*}\right)$ follows from Proposition 7 together with the fact that MCF $2 \mathrm{~b}$ contains the equations (30).

Corollary 1 The LP relaxation of formulation MCF2b satisfies the SE inequalities (7) and the GLM inequalities (8).

Our computational results (Section 5) indicate that the lower bound given by MCF 2b is signficantly stronger than the lower bounds given by either MCF1d or MCF2a. It is therefore the strongest known formulation based on commodity-flow variables.

\subsection{An alternative formulation with fewer variables}

To finish this section, we present a 'hybrid' formulation, which attempts to 'mimic' formulation MCF2b, but use only one commodity per customer, instead of two. We will show that, when a CVRP instance has symmetric costs, the lower bound from this hybrid formulation is just as good as the one from MCF $2 b$.

The basic idea of the 'hybrid' formulation is to take the formulation MCF2b (given in the Appendix), and simplify it by using aggregated flow 
variables $\tilde{f}_{i j}^{k}=f_{i j}^{k}+g_{j i}^{k}$ for all $k \in V_{c}$ and $(i, j) \in A$. (Note that these aggregated variables have no natural physical interpretation.) The constraints in the resulting formulation are as follows.

$$
\begin{aligned}
\tilde{f}^{k}\left(\delta^{+}(0)\right)=\tilde{f}^{k}\left(\delta^{-}(k)\right)=2 & \left(k \in V_{c}\right) \\
\tilde{f}^{k}\left(\delta^{-}(0)\right)=\tilde{f}^{k}\left(\delta^{+}(k)\right)=0 & \left(k \in V_{c}\right) \\
\tilde{f}^{k}\left(\delta^{+}(i)\right)=\tilde{f}^{k}\left(\delta^{-}(i)\right) & \left(k, i \in V_{c}: i \neq k\right) \\
\tilde{f}_{i j}^{k} \geq 0 & \left(i, j, k \in V_{c}: i \neq j\right) \\
\tilde{f}_{i j}^{k}+\tilde{f}_{j i}^{k} \leq x_{i j}+x_{j i} & \left(i, j, k \in V_{c}: i<j\right) \\
\sum_{k \in V_{c} \backslash\{i, j\}} q_{k}\left(\tilde{f}_{i j}^{k}+\tilde{f}_{j i}^{k}\right) \leq\left(Q-q_{i}-q_{j}\right)\left(x_{i j}+x_{j i}\right) & \left(i, j \in V_{c}: i<j\right) \\
\tilde{f}^{k}\left(\delta^{+}(i)\right)=\tilde{f}^{i}\left(\delta^{+}(k)\right) & \left(k, i \in V_{c}: i \neq k\right) .
\end{aligned}
$$

We call this formulation "MCF3". Although MCF3 is weaker than MCF2b by construction, it does have some interesting properties. These are given in the following theorem and corollaries.

Theorem 1 If the costs are symmetric, i.e. $c_{i j}=c_{j i}$ for all $(i, j) \in A$, then the lower bounds obtained by solving the LP relaxations of MCF2b and MCF3 are equal.

Proof. Let $(x, \tilde{f})$ be any feasible solution to the LP relaxation of MCF3. We can construct a feasible solution to the LP relaxation of MCF2b, say $(\bar{x}, \bar{f}, \bar{g})$, using the following mapping:

$$
\begin{aligned}
\bar{x}_{i j} & =\left(x_{i j}+x_{j i}\right) / 2 & & ((i, j) \in A) \\
\bar{f}_{i j}^{k}=\quad \bar{g}_{i j}^{k} & =\left(f_{i j}^{k}+f_{j i}^{k}\right) / 2 & & \left((i, j) \in A, k \in V_{c}\right) .
\end{aligned}
$$

Moreover, if the costs are symmetric, then the cost of $(\bar{x}, \bar{f}, \bar{g})$ is the same as that of $(x, \tilde{f})$. This shows that, when costs are symmetric, the lower bound from MCF $2 b$ cannot be better than the one from MCF3. But it cannot be worse, since MCF3 is weaker than MCF2b by construction.

Corollary 2 The LP relaxation of MCF3 satisfies the SE inequalities (7) and the GLM inequalities (8).

Proof. The in- and out-degree equations imply that $x\left(\delta^{+}(S)\right)=x\left(\delta^{-}(S)\right)$ for all $S \subset V_{c}$. It follows that the SE inequalities can be written as

$$
x\left(\delta^{+}(S)\right)+x\left(\delta^{-}(S)\right) \geq 2 \quad\left(S \subseteq V_{c}\right),
$$

and the GLM inequalities can be written as

$$
x\left(\delta^{+}(S)\right)+x\left(\delta^{-}(S)\right) \geq \frac{2}{Q} \sum_{i \in S}\left(q_{i}+\sum_{j \in V_{c} \backslash S} q_{j}\left(x_{i j}+x_{j i}\right)\right) \quad\left(S \subseteq V_{c}\right) .
$$


In this form, the inequalities are symmetric (that is, for all $(i, j) \in A$, the coefficients of $x_{i j}$ and $x_{j i}$ are equal). The result then follows from Corollary 1 and Theorem 1.

Corollary 3 The LP relaxation of MCF3 is stronger than those of SCF1, SCF2 and MCF1a.

Our computational results, given in Section 5, suggest that the LP relaxation of MCF3 may be stronger than those of MCF1b, MCF1c and MCF2a as well. On the other hand, they show that there is no dominance relationship between the LP relaxations of MCF3 and MCF1d.

\section{Stronger Bounds in Pseudo-Polynomial Time}

In this section we show that, if we are willing to sacrifice the property of having only a polynomial number of variables and constraints, then we can strengthen both MCF1d and MCF2b even further. In Subsection 4.1, we strengthen them by adding certain inequalities derived from facets of the 0-1 knapsack polytope, and show that the continuous relaxations of the resulting formulations satisfy all KLM inequalities. In Subsection 4.2, we present alternative strengthened formulations, of the same quality, which have additional variables, rather than constraints. We then show that the continuous relaxations of these latter formulations can be solved in pseudopolynomial time, via column generation.

\subsection{Valid inequalities from the knapsack polytope}

The following lemma introduces exponentially-large families of valid inequalities that, in theory, could be used to strengthen further the formulations MCF $1 d$ and MCF2b.

Lemma 3 Let $\alpha^{T} y \leq \beta$ be any valid inequality for the 0-1 knapsack polytope (10), with $\alpha \geq 0$ and $\beta>0$. Then all (integer) solutions to formulation MCF1d satisfy the inequalities

$$
\sum_{k \in V_{c} \backslash\{i\}} \alpha_{k} f_{i j}^{k} \leq\left(\beta-\alpha_{i}\right) x_{i j} \quad((i, j) \in A),
$$

and all (integer) solutions to formulation MCF2b satisfy the inequalities:

$$
\sum_{k \in V_{c} \backslash\{i, j\}} \alpha_{k}\left(f_{i j}^{k}+g_{i j}^{k}\right) \leq\left(\beta-\alpha_{i}-\alpha_{j}\right) x_{i j} \quad((i, j) \in A) .
$$

Proof. Let $(\bar{x}, \bar{f})$ be a feasible integer solution to MCF1d. If $\bar{x}_{i j}=0$, the inequality (33) holds triviallly. So, suppose that $\bar{x}_{i j}=1$, i.e., a vehicle 
traverses the arc $(i, j)$. Now consider the vector $\bar{y} \in\{0,1\}^{n}$ obtained by setting $\bar{y}_{k}$ to 1 if and only if commodity $k$ is on the vehicle just before the vehicle arrives at node $i$. Then $\bar{y}$ must be an extreme point of the polytope (10), and therefore it satisfies $\alpha^{T} y \leq \beta$. Moreover, $\bar{y}_{i}$ must equal 1 , which implies

$$
\sum_{k \in V_{c} \backslash\{i\}} \alpha_{k} \bar{y}_{k} \leq\left(\beta-\alpha_{i}\right) \bar{x}_{i j}
$$

Now, for $k \in V_{c} \backslash\{i\}$, we must have $\bar{y}_{k}=\bar{f}_{i j}^{k}$. This implies that $\bar{f}$ satisfies the inequality (33).

The proof for MCF $2 b$ is similar. The only differences are that (i) we set $\bar{y}_{k}$ to 1 if and only if commodity $k$ is on the vehicle when it leaves the depot and (ii) for $k \in V_{c} \backslash\{i, j\}$, we must have $\bar{y}_{k}=f_{i j}^{k}+g_{i j}^{k}$.

We have the following result:

Theorem 2 If all non-redundant inequalities of the form (33) are added to $M C F 1 d$, the continuous relaxation of the resulting formulation satisfies the KLM inequalities (9). The same holds if all non-redundant inequalities of the form (34) are added to MCF2b.

Proof. To show that the relaxation of MCF1d with (33) added satisfies the KLM inequalities (9), just use the proof of Proposition 2 with $\alpha_{i}$ and $\beta$ taking the place of $q_{i}$ and $Q$, and (33) taking the place of (27). To show the same result for MCF2b with (34) added, just note that the inequalities (34) are stronger than the inequalities (33).

\subsection{Alternative formulations solvable by column generation}

The models in the previous subsection have a drawback from a computational point of view. Not only are the constraints (33) and (34) exponential in number, but their coefficients are given only implicitly. To overcome this drawback, we present alternative formulations that are more explicit, but yield lower bounds of the same quality. These formulations have an exponential number of variables rather than constraints.

Let $P$ be the set of all possible loading patterns of a single vehicle. That is, each member of $P$ is a subset of $V_{c}$ whose total demand does not exceed $Q$. For each arc $(i, j)$ and for each $p \in P$, let $\lambda_{i j}^{p}$ be a binary variable, taking the value 1 if and only if a vehicle arrives at node $i$ carrying loading pattern $p$, and then traverses arc $(i, j)$. (This implies of course that $i$ and $j$ are in $p$ ). Then the model MCF1d is enlarged by adding the constraints

$$
\begin{aligned}
x_{i j} & =\sum_{p \in P: i, j \in p} \lambda_{i j}^{p} \quad\left((i, j) \in A: i, j \in V_{c}\right) \\
f_{i j}^{k} & =\sum_{p \in P: i, j, k \in p} \lambda_{i j}^{p} \quad\left((i, j) \in A: i, j \in V_{c}, k \in V_{c} \backslash\{i, j\}\right) .
\end{aligned}
$$


Now, for each arc $(i, j)$ and for each $p \in P$, let $\mu_{i j}^{p}$ be a binary variable, taking the value 1 if and only if a vehicle that departed from the depot with loading pattern $p$ goes on to traverse the arc $(i, j)$. (This implies again that $i$ and $j$ are in $p$ ). Then the model MCF2b is enlarged by adding the constraints

$$
\begin{aligned}
x_{i j} & =\sum_{p \in P: i, j \in p} \mu_{i j}^{p} \quad\left((i, j) \in A: i, j \in V_{c}\right) \\
f_{i j}^{k}+g_{i j}^{k} & =\sum_{p \in P: i, j, k \in p} \mu_{i j}^{p} \quad\left((i, j) \in A: i, j \in V_{c}, k \in V_{c} \backslash\{i, j\}\right) .
\end{aligned}
$$

Observe that the members of $P$ correspond to extreme points of the $0-1$ knapsack polytope (10). Accordingly, we call the strengthened formulations "MCF1K" and "MCF2K", respectively. The following two theorems show that these formulations have two desirable properties.

Theorem 3 The LP relaxations of formulations MCF1K and MCF2K satisfy the KLM inequalities (9).

Proof. Let $\alpha$ and $\beta$ be as in Lemma 3. From the definition of $P$ it follows that $\sum_{k \in p} \alpha_{k} \leq \beta$ for all $p \in P$. Therefore, for all $i \in V_{c}$ and all $p \in P$ such that $i \in p$, we have $\sum_{k \in p \backslash\{i\}} \alpha_{k} \leq \beta-\alpha_{i}$. This implies

$$
\sum_{p \in P: i, j \in p}\left(\sum_{k \in p \backslash\{i\}} \alpha_{k}\right) \lambda_{i j}^{p} \leq\left(\beta-\alpha_{i}\right) \sum_{p \in P: i, j \in p} \lambda_{i j}^{p}
$$

for all $(i, j) \in A$. Rearranging the left-hand side, and using (35) to simplify the right-hand side, we get

$$
\sum_{k \in V_{c} \backslash\{i\}} \alpha_{k} \sum_{p \in P: i, j, k \in p} \lambda_{i j}^{p} \leq\left(\beta-\alpha_{i}\right) x_{i j} \quad\left((i, j) \in A: i, j \in V_{c}\right) .
$$

These constraints together with (36) then imply (33). In a similar way, constraints (37) and (38) imply (34). The result then follows from Theorem 2 .

Theorem 4 The LP relaxations of MCF1K and MCF2K can be solved in pseudopolynomial time.

Proof. Suppose we have solved a restricted master problem associated with MCF1K, i.e., an LP obtained from MCF1K by relaxing the integrality condition and replacing $P$ with a small (polynomial-sized) subset $P^{\prime} \subset P$. Let $\rho_{i j}$ and $\pi_{i j}^{k}$ be the optimal dual prices for (35) and (36), respectively. For a given $(i, j) \in A$, there exists a column $\lambda_{i j}^{p}$ with negative reduced cost if and only if

$\max \left\{\sum_{k \in V_{c} \backslash\{i, j\}} \pi_{i j}^{k} y_{k}: \sum_{k \in V_{c} \backslash\{i, j\}} q_{k} y_{k} \leq Q-q_{i}-q_{j}, y \in\{0,1\}^{n-2}\right\}>\rho_{i j}$. 
This is a 0-1 knapsack problem, that can be solved in $\mathcal{O}(n Q)$ time via dynamic programming [6]. The pricing problem for MCF2K is similar. Now note that pricing in an LP is equivalent to separation in the dual of the LP. The desired results then follow from the polynomial equivalence of separation and optimization [16].

We remark that this is the first time that an LP relaxation of the CVRP has been found that can be solved in pseudo-polynomial time, yet satisfies all of the KLM inequalities. (As mentioned in Subsection 2.4, the LP relaxation of the SP formulation with elementary routes satisfies the KLM inequalities, but is strongly $\mathcal{N} \mathcal{P}$-hard to solve.) Moreover, no pseudo-polynomial separation algorithm is known for the KLM inequalities themselves.

\subsection{Comparison with set partitioning}

The strongest of our MCF formulations is MCF2K. A natural question is how the lower bound associated with MCF2K compares with the lower bound associated with the SP formulation given in Subsection 2.4. The answer to this question depends on whether or not the set $\Omega$ is permitted to contain columns that correspond to non-elementary routes. As mentioned at the end of Subsection 2.4, the LP relaxation with non-elementary routes permitted does not satisfy all KLM inequalities. Thus, the associated bound does not dominate the bound from MCF2K. The following theorem settles the question for the case in which only elementary routes are permitted:

Theorem 5 If $z^{*}$ is a solution to the LP relaxation of the SP formulation, then there exists a solution $\left(x^{*}, f^{*}, g^{*}, \mu^{*}\right)$ to the LP relaxation of MCF2K that has the same cost.

Proof. For each arc $(i, j) \in A$ and each route $r \in \Omega$, let $b_{i j r}$ be a binary constant which takes the value 1 if and only if route $r$ traverses arc $(i, j)$. Also, for any $(i, j) \in A$, any $r \in \Omega$ and any customer node $k \in V_{c}$, let $d_{i j k r}$ (respectively, $d_{i j k r}^{\prime}$ ) be a binary constant which takes the value 1 if and only if, in route $r$,

- vertex $k$ is visited and

- the arc $(i, j)$ is traversed on the way from the depot to $k$ (respectively, on the way from $k$ to the depot).

Finally, for any $(i, j) \in A, r \in \Omega$ and loading pattern $p \in P$, let $t_{i j r p}$ be a binary constant which takes the value 1 if and only if a vehicle following route $r$ departs from the depot with loading pattern $p$ and then goes on to traverse arc $(i, j)$. The desired quadruple $\left(x^{*}, f^{*}, g^{*}, \mu^{*}\right)$ is then created by setting: 
- $x_{i j}^{*}$ to $\sum_{r \in \Omega} b_{i j r} z_{r}^{*}$ for all $(i, j) \in A$;

- $f_{i j}^{* k}$ to $\sum_{r \in \Omega} d_{i j k r} z_{r}^{*}$ for all $(i, j) \in A$ and all $k \in V_{c}$;

- $g_{i j}^{* k}$ to $\sum_{r \in \Omega} d_{i j k r}^{\prime} z_{r}^{*}$ for all $(i, j) \in A$ and all $k \in V_{c}$;

- $\mu_{i j}^{* p}$ to $\sum_{r \in \Omega} t_{i j r p} z_{r}^{*}$ for all $(i, j) \in A$ and $p \in P$.

This quadruple has the same cost as $z^{*}$, from the definition of $c_{r}$ in the objective of the SP formulation. One can check that it also satisfies all of the linear constraints in the formulation MCF2K, i.e., the constraints (2), (3), (13)-(16), (20)-(24), (31), (30), (37) and (38).

Theorem 5 implies that the lower bound from MCF2K is dominated by the lower bound from the SP formulation with elementary routes. We stress however that the former bound can be computed in pseudo-polynomial time, whereas the latter bound cannot (unless $\mathcal{P}=\mathcal{N} \mathcal{P}$ ).

\section{Computational Experiments}

In this section, we report on some computational experiments. We stress from the outset that the goal of these experiments was not to solve large-scale CVRP instances to proven optimality, but rather to establish dominance relations between the lower bounds obtained when solving the LP relaxation of various formulations. (The development of a viable exact algorithm for the CVRP based on formulation MCF2K may be the topic of a future paper.) We found that, for this purpose, it was sufficient to use small instances with $n=16$. The advantage of using these instances is that the $\mathrm{RC}$ inequalities (4) can be enumerated and added to the LP relaxation if desired. (No efficient separation procedure is known for the $\mathrm{RC}$ inequalities.)

We created both asymmetric and symmetric instances. In the asymmetric instances, the costs $c_{i j}$ were randomly generated in $[0,500]$. In the symmetric instances, the $\operatorname{costs} c_{i j}$ were obtained by computing the Euclidean distance between locations randomly distributed in the square $[0,500] \times[0,500]$, except the depot, which was located in the center of the square. We created instances with general demands (random integers in the range $[25,33]$ ) and instances with only unit demands. For the instances with general demands, we considered $Q \in\{100,150,200\}$. For the instances with unit demands, we considered $Q \in\{4,6,8\}$. This led to twelve families of instances, and for each family we generated 20 instances. The instance generator and the formulations were implemented in FICO Xpress Mosel 3, and the source code is available to readers on request to the authors.

Table 1 gives the results for seven LP relaxations that only involve $x$ variables. The first column describes the instance type, and the remaining 
Table 1: Average ratios for relaxations in $x$-space.

\begin{tabular}{cccccccc}
\hline Type & SE & FC & GLM & RC & FC+SE & GLM+SE & GLM+RC \\
\hline A-G-100 & 42.47 & 74.76 & 80.09 & 91.61 & 74.96 & 80.10 & 91.82 \\
A-G-150 & 64.10 & 82.56 & 84.57 & 89.88 & 83.03 & 84.77 & 89.88 \\
A-G-200 & 72.64 & 81.35 & 82.14 & 96.62 & 82.17 & 82.70 & 96.62 \\
\hline A-U-4 & 54.37 & 83.99 & 87.92 & 94.30 & 84.33 & 87.92 & 94.35 \\
A-U-6 & 72.12 & 85.85 & 87.34 & 97.65 & 86.62 & 87.79 & 97.65 \\
A-U-8 & 87.37 & 93.76 & 94.33 & 98.05 & 94.98 & 95.25 & 98.05 \\
\hline \hline S-G-100 & 61.53 & 81.52 & 87.48 & 98.70 & 85.42 & 89.65 & 98.72 \\
S-G-150 & 76.83 & 82.85 & 86.91 & 99.62 & 90.95 & 92.95 & 99.62 \\
S-G-200 & 82.31 & 79.70 & 82.07 & 99.64 & 90.25 & 91.38 & 99.64 \\
\hline S-U-4 & 70.01 & 85.20 & 90.76 & 99.98 & 90.66 & 94.28 & 99.99 \\
S-U-6 & 81.17 & 82.43 & 85.43 & 99.95 & 91.94 & 93.45 & 99.95 \\
S-U-8 & 89.51 & 82.84 & 84.71 & 100.00 & 95.44 & 96.14 & 100.00 \\
\hline
\end{tabular}

columns summarise the results that we obtained when adding various combinations of the SE, FC, GLM and RC inequalities to the LP relaxation consisting of (1)-(3) and non-negativity. Each figure is the average, over 20 instances, of the ratio between the lower bound and the optimum, expressed as a percentage. Table 2 gives analogous results for six LP relaxations that involve $f$-variables, Table 3 does the same for five relaxations that involve $f$ - and $g$-variables, and Table 4 does the same for four SP relaxations, that involve $x$ and $z$ variables. Columns nSP and eSP refer to the SP relaxation with non-elementary and elementary routes, respectively. Columns nSP+RC and $\mathrm{eSP}+\mathrm{RC}$ refer to the same relaxations strengthened with $\mathrm{RC}$ inequalities (4).

The main conclusion from the results in Table 1 is that the RC inequalities are the most important inequalities by far. Comparing Tables 1 and 2, we see that, as expected, MCF1a gives the same bound as the FC inequalities. We also see that the four new formulations give better bounds than MCF 1a and MCF 1b, and also better bounds than the SE and GLM inequalities combined. Note also that there is no dominance between MCF1a and MCF1b, but MCF $1 \mathrm{~b}$ tends to perform poorly. Moreover, as expected, MCF1c, MCF1d and MCF $1 \mathrm{~K}$ are of increasing strength. As for MCF3, we see that it appears to dominate MCF1c, but does not dominate MCF1d. Interestingly, MCF1K does not dominate MCF3, despite the fact that it satisfies all KLM inequalities and is weakly $\mathcal{N} \mathcal{P}$-hard to compute. Also, MCF1K gives the same bound as MCF1d in the unit demand case.

Turning our attention to Table 3 , we see that MCF2a consistently gives worse bounds than the SE and GLM inequalities combined. As expected, MCF $2 b$ dominates MCF1d and MCF2a, and gives the same bound as MCF3 on the 
Table 2: Average ratios for relaxations in $(x, f)$-space.

\begin{tabular}{ccccccc}
\hline Type & MCF1a & MCF1b & MCF1c & MCF1d & MCF1K & MCF3 \\
\hline A-G-100 & 74.76 & 51.18 & 80.99 & 82.34 & 87.45 & 81.99 \\
A-G-150 & 82.56 & 68.62 & 85.44 & 86.25 & 86.90 & 86.17 \\
A-G-200 & 81.35 & 75.14 & 83.18 & 83.54 & 83.93 & 83.53 \\
\hline A-U-4 & 83.99 & 61.89 & 88.77 & 89.98 & 89.98 & 90.04 \\
A-U-6 & 85.85 & 75.65 & 88.36 & 88.88 & 88.88 & 88.93 \\
A-U-8 & 93.76 & 89.61 & 95.53 & 95.82 & 95.82 & 95.86 \\
\hline \hline S-G-100 & 81.52 & 65.99 & 89.84 & 90.06 & 91.09 & 91.79 \\
S-G-150 & 82.85 & 78.30 & 93.03 & 93.27 & 93.66 & 95.01 \\
S-G-200 & 79.70 & 82.74 & 91.47 & 91.60 & 91.93 & 93.02 \\
\hline S-U-4 & 85.20 & 73.28 & 94.37 & 94.64 & 94.64 & 96.73 \\
S-U-6 & 82.43 & 82.00 & 93.55 & 93.74 & 93.74 & 95.33 \\
S-U-8 & 82.84 & 89.51 & 96.21 & 96.33 & 96.33 & 97.83 \\
\hline
\end{tabular}

Table 3: Average ratios for relaxations in $(x, f, g)$-space.

\begin{tabular}{cccccc}
\hline Type & MCF2a & MCF2b & MCF2K & MCF2b+RC & MCF2K+RC \\
\hline A-G-100 & 76.62 & 85.68 & 96.97 & 93.61 & 98.00 \\
A-G-150 & 80.25 & 88.86 & 91.68 & 91.15 & 92.30 \\
A-G-200 & 79.46 & 85.16 & 87.10 & 96.89 & 97.14 \\
\hline A-U-4 & 83.57 & 93.43 & 93.43 & 96.34 & 96.34 \\
A-U-6 & 83.36 & 91.16 & 91.16 & 98.04 & 98.04 \\
A-U-8 & 92.39 & 97.10 & 97.10 & 98.38 & 98.38 \\
\hline \hline S-G-100 & 80.61 & 91.79 & 93.94 & 98.74 & 99.08 \\
S-G-150 & 86.92 & 95.01 & 95.99 & 99.64 & 99.67 \\
S-G-200 & 87.09 & 93.02 & 93.85 & 99.64 & 99.70 \\
\hline S-U-4 & 85.99 & 96.73 & 96.73 & 100.00 & 100.00 \\
S-U-6 & 88.25 & 95.33 & 95.33 & 99.96 & 99.96 \\
S-U-8 & 92.44 & 97.83 & 97.83 & 100.00 & 100.00 \\
\hline
\end{tabular}


Table 4: Average ratios for relaxations in $(x, z)$-space.

\begin{tabular}{ccccc}
\hline Type & nSP & nSP + RC & eSP & eSP+RC \\
\hline A-G-100 & 98.56 & 98.72 & 98.59 & 98.72 \\
A-G-150 & 93.40 & 93.76 & 94.09 & 94.25 \\
A-G-200 & 89.38 & 97.45 & 90.94 & 97.67 \\
\hline A-U-4 & 94.76 & 96.86 & 94.97 & 96.92 \\
A-U-6 & 92.11 & 98.19 & 93.39 & 98.40 \\
A-U-8 & 96.97 & 98.28 & 98.43 & 98.86 \\
\hline \hline S-G-100 & 95.05 & 99.39 & 98.46 & 99.76 \\
S-G-150 & 90.78 & 99.67 & 98.69 & 99.88 \\
S-G-200 & 85.37 & 99.70 & 97.16 & 99.83 \\
\hline S-U-4 & 91.91 & 100.00 & 98.29 & 100.00 \\
S-U-6 & 86.66 & 99.95 & 97.27 & 99.99 \\
S-U-8 & 85.74 & 100.00 & 99.50 & 100.00 \\
\hline
\end{tabular}

symmetric instances. Also as expected, MCF2K dominates MCF1K and MCF2b. In fact, it is significantly stronger than MCF1K in all cases. On the other hand, MCF2K gives the same bound as MCF2b in the unit demand case. Note also that using MCF $2 b$ or MCF2K in combination with the RC inequalities gives better results than using RC and GLM inequalities in combination. The difference is noticeable especially for the asymmetric instances.

Now consider Table 4. We see that, as expected, the SP relaxation with non-elementary routes dominates the GLM relaxation. On the other hand, it is weaker even than MCF1c in some cases, despite being weakly $\mathcal{N} \mathcal{P}$-hard to compute. Moreover, it neither dominates nor is dominated by MCF2K, which is also weakly $\mathcal{N} \mathcal{P}$-hard to compute. As for the SP relaxation with elementary routes, which is strongly $\mathcal{N} \mathcal{P}$-hard to compute, it dominates all of the MCF formulations. This is in accordance with Theorem 5. Finally, including RC inequalities makes a considerable difference in some cases.

To aid the reader, Figure 1 shows all known dominance relations between all relaxations considered. An arrow from one relaxation to another indicates that the latter is stronger than the former.

\section{Conclusion}

In this paper, we have surveyed the known CVRP formulations based on additional commodity-flow variables, and introduced several new multicommodity flow formulations that are provably stronger than all of them, in both theory and practice. Of particular interest is the formulation that we have called MCF2b, which is of polynomial size and yields lower bounds 
Figure 1: Dominance between formulations.

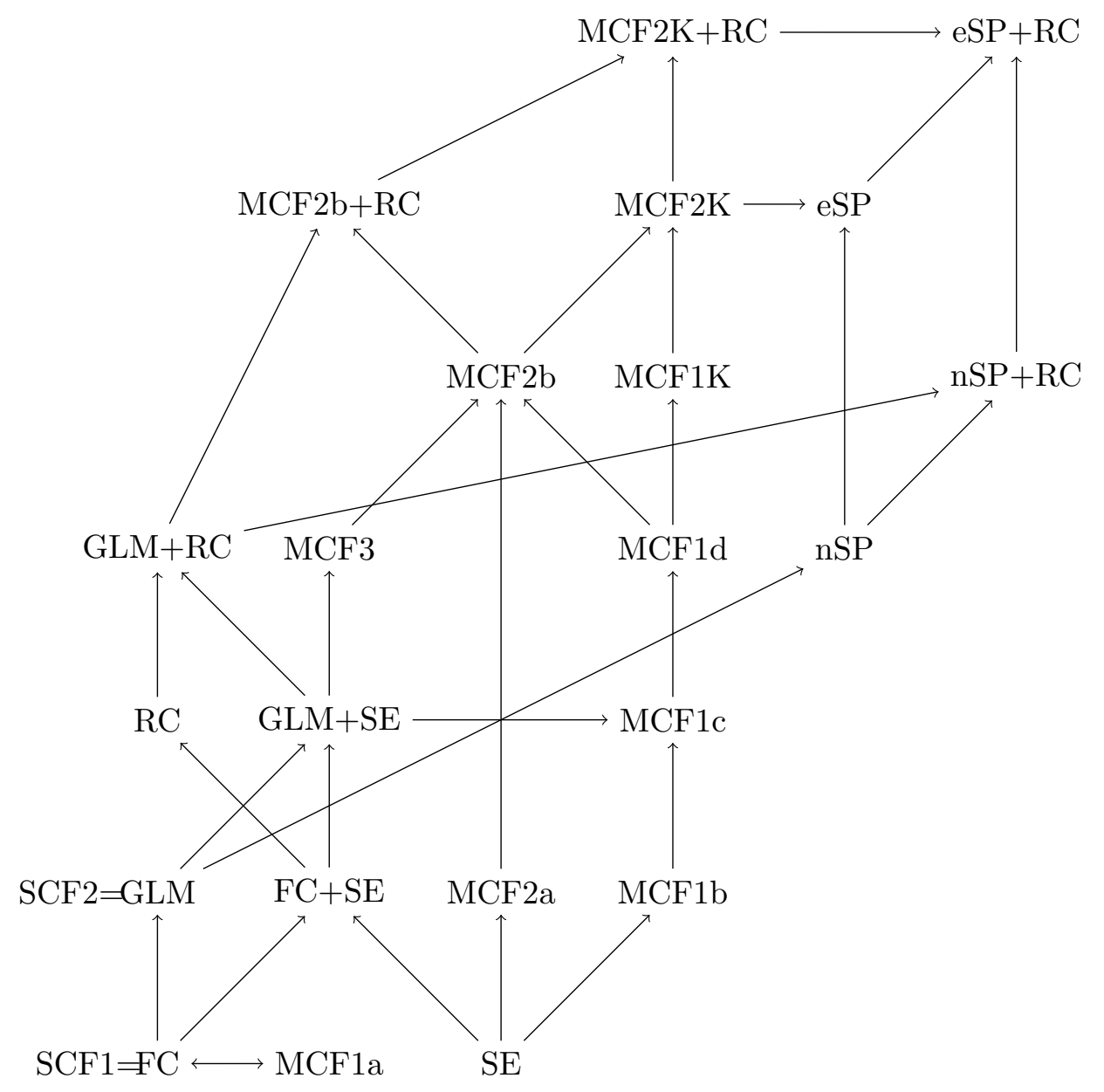


that are significantly stronger than those obtained by all other known formulations of polynomial size.

A natural question for future research is whether one could devise a competitive exact algorithm for the CVRP based upon one of our new formulations, or some similar formulation. To do this, some kind of decomposition scheme might be needed. Another interesting research topic would be to completely characterise the projections into $x$-space of the feasible regions of the LP relaxations of MCF1b to MCF1d, MCF2a and MCF2b, and possibly devise efficient separation routines for them, so that the additional flow variables could be avoided. Finally, one could attempt to adapt our formulations to other similar vehicle routing problems, such as the CVRP with pickup-and-delivery [8], the multi-commodity TSP described in [17], or the VRP with load-dependent costs [19].

Acknowledgement: The second author was supported by the research project MTM2012-36163-C06-01.

\section{References}

[1] Agarwal, Y., Mathur, K., Salkin, H.M. (1989) A set-partitioning-based exact algorithm for the vehicle routing problem. Networks 19 731-749 (1989)

[2] Baldacci, R., Hadjiconstantinou, E., Mingozzi, A.: An exact algorithm for the capacitated vehicle routing problem based on a two-commodity network flow formulation. Oper. Res. 52 723-738 (2004)

[3] Baldacci, R., Christofides, N., Mingozzi, A.: An exact algorithm for the vehicle routing problem based on the set partitioning formulation with additional cuts. Math. Program. 115 351-385 (2008)

[4] Balinski, M.L., Quandt, R.E.: On an integer program for a delivery problem. Oper. Res. 12 300-304 (1964)

[5] Ball, M., Magnanti, T.L., Monma, C.L., Nemhauser, G.L. (eds.) Network Routing. Handbooks in Operations Research and Management Science, vol. 8. Amsterdam: North Holland (1995)

[6] Bellman, R.E.: Dynamic Programming. Princeton, NJ: Princeton University Press (1957)

[7] Dantzig, G.B., Ramser, R.H.: The truck dispatching problem. Mngt. Sci. 6, 80-91 (1959)

[8] Desaulniers, G., Desrosiers, J., Erdmann, A., Solomon, M.M., Soumis, F.: VRP with pickup and delivery. In Toth, P., Vigo D. (eds.): The Vehicle Routing Problem. SIAM (2001) 
[9] Foster, B.A., Ryan, D.M.: An integer programming approach to the vehicle scheduling problem. Oper. Res. Quart. 27 367-384 (1976)

[10] Fukasawa, R., Lysgaard, J., de Aragão, M.P., Reis, M., Uchoa, E., Werneck, R.F.: Robust branch-and-cut-and-price for the capacitated vehicle routing problem. Math. Program. 106 491-511 (2006)

[11] Garvin, W.W., Crandall, H.W., John, J.B., Spellman, R.A.: Applications of linear programming in the oil industry. Mgmt. Sci. 3 407-430 (1957)

[12] Gavish, B., Graves, S.: The traveling salesman problem and related problems. Working Paper, Graduate School of Management, University of Rochester, New York (1979)

[13] Gavish, B.: The delivery problem: new cutting planes procedures. Presented at the TIMS XXVI conference, Copenhagen 1984.

[14] Golden, B.L., Raghavan, S., Wasil, E.A. (eds.): The Vehicle Routing Problem: Latest Advances and New Challenges. New York: Springer (2008)

[15] Gouveia, L. A result on projection for the vehicle routing problem. Eur. J. Oper. Res. 85 610-624 (1995)

[16] Grötschel, M., Lovász, L., Schrijver, A.: The ellipsoid method and its consequences in combinatorial optimization. Combinatorica 1 169-197 (1981)

[17] Hernández-Pérez, H., Salazar-González, J.J.: The multi-commodity one-to-one pickup-and-delivery traveling salesman problem. Eur. J. Oper. Res. 196 987-995 (2009)

[18] Hoffman, A.J.: Some recent applications of the theory of linear inequalities to extremal combinatorial analysis. In Bellman, R.,Hall, M. (eds.) Proceedings of Symposia in Applied Mathematics, Vol. X: Combinatorial Analysis. Providence, RI: AMS (1960)

[19] Kara, I., Kara, B.Y., Yetis, M.K.: Energy minimizing vehicle routing problem. In Dress, A., Xu, Y., Zhu, B. (eds.) Combinatorial Optimization and Applications, pp. 62-71. Lecture Notes in Computer Science, vol. 4616, Springer: Berlin-Heidelberg (2007)

[20] Laporte, G., Nobert, Y.: A branch and bound algorithm for the capacitated vehicle routing problem. OR Spektrum 5 77-85 (1983)

[21] Letchford, A.N., Eglese, R.W., Lysgaard, J.: Multistars, partial multistars and the capacitated vehicle routing problem. Math. Program. 94 21-40 (2002) 
[22] Letchford, A.N., Salazar-González, J.J.: Projection results for vehicle routing. Math. Program. 105 251-274 (2006)

[23] Lysgaard, J., Letchford, A.N., Eglese, R.W.: A new branch-and-cut algorithm for the capacitated vehicle routing problem. Math. Program. $100423-445$ (2004)

[24] Martinelli, R., Pecin, D., Poggi, M.: Efficient elementary and restricted non-elementary route pricing. Eur. J. Oper. Res. 239 102-111 (2014)

[25] Naddef, D., Rinaldi, G.: Branch-and-cut algorithms for the capacitated VRP. In Toth, P., Vigo, D. (eds.): The Vehicle Routing Problem. SIAM (2001)

[26] Papernov, B.A.: On existence of multicommodity flows (in Russian). In Fridman, A.A. (ed.) Studies in Discrete Optimization. Moscow: Nauka (1976)

[27] Toth, P., Vigo, D. (eds.): The Vehicle Routing Problem. SIAM (2001) 


\section{Appendix}

For ease of reference, we include here two of our multi-commodity flow formulations: MCF1d, which is the strongest known formulation of polynomial size that involves only $x$ and $f$ variables, and MCF2b, which is the strongest known formulation of polynomial size that involves $x, f$ and $g$ variables.

\section{Formulation MCF1d:}

$$
\begin{aligned}
& \min \sum_{(i, j) \in A} c_{i j} x_{i j} \\
& \text { s.t. } \quad x\left(\delta^{+}(i)\right)=x\left(\delta^{-}(i)\right)=1 \quad\left(i \in V_{c}\right) \\
& x_{i j} \in\{0,1\} \quad((i, j) \in A) \\
& f^{k}\left(\delta^{+}(0)\right)=f^{k}\left(\delta^{-}(k)\right)=1 \quad\left(k \in V_{c}\right) \\
& f^{k}\left(\delta^{-}(0)\right)=f^{k}\left(\delta^{+}(k)\right)=0 \quad\left(k \in V_{c}\right) \\
& f^{k}\left(\delta^{+}(i)\right)=f^{k}\left(\delta^{-}(i)\right) \quad\left(k, i \in V_{c}: i \neq k\right) \\
& 0 \leq f_{i j}^{k} \leq x_{i j} \quad\left(k \in V_{c},(i, j) \in A\right) \\
& \sum_{k \in V_{c} \backslash\{i, j\}} q_{k} f_{i j}^{k} \leq\left(Q-q_{i}-q_{j}\right) x_{i j} \quad((i, j) \in A) \\
& \sum_{i \in V_{c} \backslash\{k\}} q_{i}\left(f^{k}\left(\delta^{+}(i)\right)+f^{i}\left(\delta^{+}(k)\right)\right) \leq Q-q_{k} \quad\left(k \in V_{c}\right) .
\end{aligned}
$$

\section{Formulation MCF2b:}

$$
\begin{array}{cl}
\min \sum_{(i, j) \in A} c_{i j} x_{i j} & \\
\text { s.t. } \quad x\left(\delta^{+}(i)\right)=x\left(\delta^{-}(i)\right)=1 & \left(i \in V_{c}\right) \\
x_{i j} \in\{0,1\} & ((i, j) \in A) \\
f^{k}\left(\delta^{+}(0)\right)=f^{k}\left(\delta^{-}(k)\right)=g^{k}\left(\delta^{+}(k)\right)=g^{k}\left(\delta^{-}(0)\right)=1 & \left(k \in V_{c}\right) \\
f^{k}\left(\delta^{-}(0)\right)=f^{k}\left(\delta^{+}(k)\right)=g^{k}\left(\delta^{-}(k)\right)=g^{k}\left(\delta^{+}(0)\right)=0 & \left(k \in V_{c}\right) \\
f^{k}\left(\delta^{+}(i)\right)=f^{k}\left(\delta^{-}(i)\right)=g^{i}\left(\delta^{+}(k)\right)=g^{i}\left(\delta^{-}(k)\right) & \left(k, i \in V_{c}: i \neq k\right) \\
\sum_{i j}^{k}, g_{i j}^{k} \geq 0 \quad ; \quad f_{i j}^{k}+g_{i j}^{k} \leq x_{i j} & \left(k \in V_{c},(i, j) \in A\right) \\
\sum_{k \in V_{c} \backslash\{i, j\}} q_{k}\left(f_{i j}^{k}+g_{i j}^{k}\right) \leq\left(Q-q_{i}-q_{j}\right) x_{i j} & ((i, j) \in A) .
\end{array}
$$

